\title{
DISCOVERY OF NON-RADIAL PULSATIONS IN THE WHITE DWARF PRIMARY OF A CATACLYSMIC VARIABLE STAR
}

\author{
BRIAN WARNER AND LIZA VAN ZYL \\ Department of Astronomy \\ University of Cape Town, Rondebosch 7700. South Africa
}

\section{Introduction}

Non-radial pulsations in isolated white dwarfs have been known for 25 years and it has been shown that the hydrogen-rich (DA) white dwarfs have a high probability of pulsating if they lie in the instability strip with effective temperature between 11500 and $13200 \mathrm{~K}$ - the ZZ Ceti stars (e.g. Kepler and Nelan 1993). Analysing techniques developed for such stars allow derivation of masses, luminosities, rotation periods, hydrogen surface layer masses and other properties (e.g. Kepler and Bradley 1995). A number of binary systems are known in which the primary is a white dwarf; dominant in this class are the cataclysmic variable (CV) stars. Until now no CV primary has been found to have non-radial pulsations.

GW Lib had an outburst of large amplitude in 1983 and was thought to be a nova. Spectra at minimum light (mag 18.5) show it to be in fact a dwarf nova (Duerbeck and Seitter 1987). No other outbursts have been observed, so it is deduced to be a $\mathrm{CV}$ of very low rate of mass transfer and long outburst interval (i.e., an extreme SU UMa star), similar to WZ Sge or AL Com. Our observations of GW Lib in March and April 1997, using the University of Cape Town CCD photometer on the 40in reflector at the Sutherland site of the South African Astronomical Observatory, with integrations (in white light) down to 6 secs, show the presence of multiperiodic oscillations. In the March observations coherent periods at 378.72 and 648.07 secs were present throughout the week. In April these were present at greatly reduced amplitude and other oscillation modes with periods near $390,400,680$, and also probably at 890 , 1020 and 1080 secs had become visible. During the latter week there was considerable variation of amplitude of the various modes.

These periods and amplitude variations are characteristic of the larger amplitude ZZ Ceti stars like G29-38 and G38-29 (Kleinman 1995). In GW Lib the typical amplitude is $3 \%$, but about half of the light in the visible region comes from accretionrelated luminosity; the true amplitude is therefore about twice what is observed. A periodic signal near $119 \mathrm{~min}$, with a harmonic at half this period, was also occasionally seen, which almost certainly is modulation at the orbital period, and is compatible with the expectation that the outburst characteristics imply SU UMa behaviour (e.g. Warner 1995). The presence of a rich spectrum of non-radial oscillation modes in GW Lib should eventually lead to the first really accurate determination of the physical 


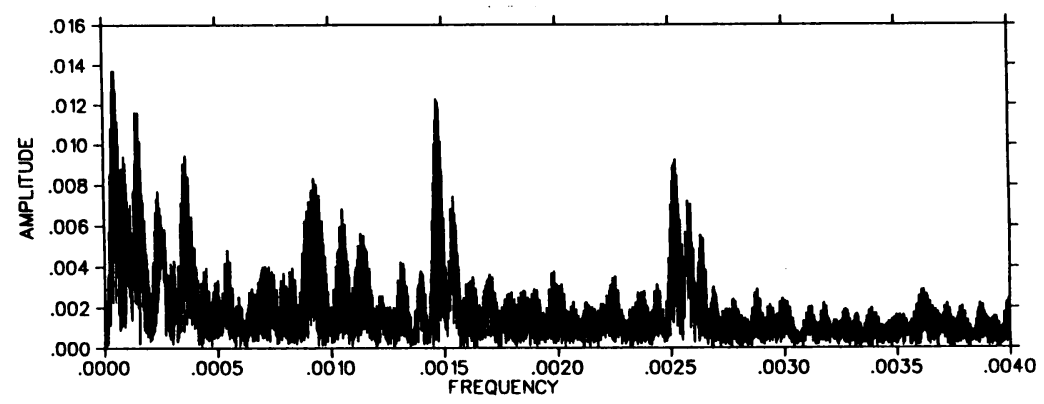

Figure 1. Amplitude spectrum of 4 nights of observations of GW Lib acquired in April 1997

properties of a CV primary - which will be valuable as a standard against which to test the more conventional spectroscopic and photometric methods of determining masses of the primaries. The primaries in CVs have a wide range of surface temperatures (see Table 2.8 of Warner 1995), which, as almost all CV primaries are old enough to have cooled to below $9000 \mathrm{~K}$, probably shows that it is the average accretion rate that determines the surface temperature (see p. 478 of Warner 1995). To be in the instability strip (at least, as found in single stars) a CV primary must have just the low rate of mass transfer that ensures that it will be a very long interval dwarf nova. Observations of the changes in oscillation frequency and amplitude after the next superoutburst will inform studies of non-radial oscillations and also models of structural changes that occur during accretion events.

There is another long-interval SU UMa star which may have non-radial oscillations; this is SW UMa, which at quiescence has a light curve similar to that of GW $\mathrm{Lib}$ and in which the dominant period is 950 secs (Shafter et al. 1986). WZ Sge has been thoroughly observed and exhibits modulations near 29 secs which are probably related to the rotation of the primary. Its $\mathrm{T}_{\text {eff }}$ of $14900 \mathrm{~K}$ (Sion et al. 1995) places it outside the (isolated) white dwarf instability strip. If SW UMa does turn out to have non-radial oscillations it would be of great interest to determine its $\mathrm{T}_{\text {eff }}$, and that of GW Lib, and compare them with WZ Sge.

\section{References}

Clemens, J.C., 1993. Baltic Astr. 2, 407.

Duerbeck, H.W. and Seitter, W.C., 1987. Astrophys. Sp. Sci. 131, 467.

Kepler, S.O. and Bradley, P.A., 1995. Baltic Astr. 4, 166.

Kleinman, S.J., 1995. Baltic Astr. 4, 270.

Shafter, A.W., Szkody, P. \& Thorstensen, J., Astrophys. J. 308, 765.

Sion, E.M. et al., 1995. Astrophys. J. 439, 957.

Warner, B., 1995. Cataclysmic Variable Stars, Cambridge University Press, Cambridge. 